\title{
¿QUIÉN ES FRANCIS FUKUYAMA?
}

\author{
Por \\ ISRAEL SANMARTÍN ${ }^{1}$
}

\begin{abstract}
Además de ser autor del famoso artículo The end of History? ${ }^{2}$ y del no menos célebre libro The end of History and the last man $^{3}$, ¿a que tradición intelectual pertenece Fukuyama? ¿Cuál es su formación académica? ¿En qué dirección investigó antes de escribir su famosa teoría teleológica? ¿En que contextos surge su famosa tesis?, en definitiva, ¿quién es Francis Fukuyama? Debido a los diferentes artículos que se han escrito sobre el tema deberían de haber quedado aclaradas algunas de estas interrogantes. Sin embargo, la reciente polémica suscitada por su nuevo ensayo «The end of order» ha devuelto a Fukuyama a la actualidad intelectual y han aflorado algunas de las lagunas existentes entorno a su figura. Como muestra, dos artículos recientes aparecidos en el diario $E l M u n d o^{4}$ califi-
\end{abstract}

\footnotetext{
${ }^{1}$ Este trabajo está realizado dentro del marco del proyecto de investigación «El Estado de la historia», realizado en el Instituto de Estudios Gallegos «Padre Sarmiento» (CSIC) bajo la dirección del doctor C. Barros y financiado por la Xunta de Galicia (XUGA 40101B96).

${ }^{2}$ FUKUYAMA, F., «The end of History?», The National Interest, Washington, $\mathrm{n}^{\circ} 16$, summer 1989, pp. 3-18. Traducción en España: FUKUYAMA, F., «¿El final de la Historia?, EL País, Madrid, 24 de septiembre, 1989, y FUKUYAMA, F, ¿El final de la Historia?», Claves de Razón Práctica, Madrid, nº 1, Abril 1990, pp. 85-96.

${ }^{3}$ The Free Press, New York, 1992. Traducción española FUKUYAMA, F., El fin de la Historia y el último hombre, trad. P. Elías, Planeta, Barcelona, 1992.

${ }^{4}$ VIDAL, J. M., «La culpa es de ellas, según Fukuyama», suplemento Crónica, El Mundo, 23 de noviembre de 1997, p. 11; ALTICHIERI, Alessio, «Francis Fukuyama. El filósofo de la misoginia», suplemento La Esfera, El Mundo, 29 de noviembre de 1997, p. 6 .
}

"CUADERNOS DE ESTUDIOS GALLEGOS", Tomo XLVI, Fascículo 111, Santiago 1999. 
can a Fukuyama como «historiador americano de origen japonés» ${ }^{5} \mathrm{y}$ "polémico historiador americano» ${ }^{6}$. Ante esta confusión resulta pertinente matizar algunas cuestiones y revelar algunos datos que ayuden a comprender mejor la obra del intelectual norteamericano. A ello están dedicadas las siguientes líneas.

Recientemente, el hispanista Geoffrey Parker ${ }^{7}$ declaraba en la prensa que los norteamericanos tienen miedo a perder su imperio y están preocupados de que su supremacía desaparezca en algún momento ${ }^{8}$. En un contexto similar, apareció la tesis de «el final de la Historia» de Francis Fukuyama, quien alientado por diferentes mentores concibió su teoría con la intencionalidad política de legitimar la democracia liberal y con el propósito de universalizar el sistema económico capitalista. Esta defensa del sistema político de EE. UU. y parte del mundo occidental se anticipó al derrumbamiento de las economías planificadas de la U. R. S. S. y los demás países del Este, adelantando la tesis a la caída y dejando al norteamericano como una especie de profeta. Coincidencias históricas aparte, «el fin de la Historia» pertenece al amplio elenco de los trabajos de encargo, y conlleva, la defensa del sistema liberal y la defenestración, al precio que sea, del sistema comunista y del marxismo.

CONTEXTO POLÍTICO. Por otro lado, es interesante ver el contexto político en el que surgía la tesis de Fukuyama. En las elecciones de 1988, George Bush, después de una campaña presidencial destacada por las profundidades demagógicas y la falta de escrúpulos ${ }^{9}$, tomaba el relevo de Ronald Reagan en el Partido Republicano de los EE. UU. De esta forma,

${ }^{5}$ VIDAL, J. M., «La culpa es de ellas, según Fukuyama», p. 11.

${ }^{6}$ ALTICHIERI, A., «Francis Fukuyama. El filósofo de la misoginia», p. 6.

${ }^{7}$ Geoffrey Parker es un historiador de origen inglés nacionalizado norteamericano. Desde 1993 imparte sus clases en la Universidad de Yale, donde es profesor de Historia Militar y Naval.

${ }^{8}$ Estas declaraciones aparecen en el diario $A b c, 9$ de julio, 1996, p. 63.

${ }^{9}$ MILIBAND, Ralph «Reflexiones sobre la crisis de los regímenes comunistas», trad. Ana Ferrero y Mercedes Villegas, en Robin Blackburn ed., Después de la caída. El fracaso del comunismo y el futuro del socialismo, Editorial Crítica, Barcelona, 1993, p. 37. (After the fall. The failure of communism and the future of socialism, Verso, London, 1991).

"CUADERNOS DE ESTUDIOS GALLEGOS", Tomo XLVI, Fascículo 111, Santiago 1999. 
el neoliberalismo ${ }^{10}$, ideología defensora de la reducción del estatismo y auge de la privatización, proseguía su andadura en el marco de la historia contemporánea. Bush, antiguo director de la CIA y jefe de la lucha antidroga para la administración Reagan ${ }^{11}$ era elegido presidente de los Estados Unidos por una gran mayoría el 8 de noviembre de 1988. El 20 de enero de 1989 tomaba posesión como presidente del país norteamericano. En sus cuatro años de mandato, Bush recuperó la peor cara de la política exterior beligerante e intervencionista del presidente Reagan, en la guerra contra Irak desarrollada en el Golfo Pérsico en 1991 (pese a que antes había apoyado la invasión de Irak a Irán ${ }^{12}$ ), los intentos de reducción del poder árabe sobre Palestina y la invasión de Panamá, entre otras.

En otro orden de cosas, Bush fue contemporáneo de Gorbachov y ambos asistieron al final de la «guerra fría». Los dos mantuvieron relaciones cordiales, puesto que Estados Unidos, al igual que todas las democracias

${ }^{10}$ El neoliberalismo es un fenómeno diferente del liberalismo clásico del siglo pasado. El neoliberalismo nació después de la segunda guerra mundial en Inglaterra y Estados Unidos. El texto de Friedrich Hayek Camino a la servidumbre escrito en 1944, junto con diferentes escritos de Raymond Aron, Isaiah Berlin y John Rawls, fueron la base de esa ideología. En principio, constituyó una reacción contra el Partido Laborista Inglés y el New Deal norteamericano. Era una reacción teórica y política contra el Estado intervencionista y de bienestar. En 1947 Hayek promovería una reunión con quienes compartían su visión ideológica, y fueron: Milton Friedman, Karl Popper, Lionel Robbins, Ludwing Von Mises, Walter Eukpen, Walter Lippman, Michael Polanyi y Salvador de Madariaga. Los años 80 fueron los años del triunfo de la ideología neoliberal con Margaret Thatcher en Inglaterra, Hetmun Khol en Alemania y Ronald Reagan en Estados Unidos. Para Perry Anderson se habría producido un nuevo brote de neoliberalismo, con un desprecio absoluto por el Keynessianismo y el Estado de bienestar en las economías poscomunistas del Este (Polonia, Rusia, República Checa) y en América Latina (Bolivia, Chile, México, Argentina, Venezuela y Perú). Ver ANDERSON, Perry, «Neoliberalismo: Balance y perspectivas para la izquierda», Viento Sur, n 26, mayo 1996, pp. 107-120; VALLESPÍN, Fernando y GARCÍA- GUITIÁN, Elena, «El neoliberalismo (1): Friedrich Hayek, Raymond Aron e Isaiah Berlín», en Fernando Vallespín, Historia de la Teoría Política, vol. 6, Alianza Editorial, Madrid, 1995, pp. 15- 87; BELTRÁN PEDREIRA, Elena, «El neoliberalismo (2): la Filosofía Política de John Rawls», en Fernando Vallespín, Historia de la Teoría Política, Alianza editorial, Madrid, 1995, pp. 88- 150.

${ }^{11}$ CHOMSKY, Noam, El miedo a la democracia, trad. Miraeia Carol, Editorial Crítica, Barcelona, 1992 (Deterring democracy, New York, 1991), p. 193.

${ }^{12}$ CHOMSKY, Noam, El miedo a la democracia, p. 236. 
occidentales, querían el mantenimiento de Gorbachov ${ }^{13}$. Pese a esto, Bush fue testigo de la caída de Gorbachov y su «perestroika».

CONTEXTO INTELECTUAL. Hay otro contexto donde surgía Fukuyama: el intelectual. El autor de El final de la Historia y el último hombre es heredero de una larga tradición de pensamiento conservador, denominado en Estados Unidos «neocons». Durante años, los conservadores fueron apartados de la vida intelectual estadounidense. Un primer intento de izar la bandera del conservadurismo lo sostuvo Russell Kirk con su libro The conservative Mind ${ }^{14}$, donde ensalzaba la creencia en el orden, la tradición, la jerarquía y la autoridad. Pero no fue hasta la irrupción de la revista The National Review cuando cristalizó el conservadurismo. Esa revista, dirigida por William F. Buckley funcionaba en base a un consenso político anticomunista.

Sin embargo no sería hasta la década de los setenta cuando surgirían los «neocons» ${ }^{15}$. Este grupo de intelectuales le dio al conservadurismo norteamericano un nuevo vocabulario y una nueva sensibilidad. Dentro de este grupo se encontraban: Nisbet, Bell, Kristol, Glazer, Novak, Podhoretz, Lipset, Shils, Wildousky, Huntington, Kickpatrick, Brezinsky, Starr, Wilson y Banfield ${ }^{16}$. Todos se apoyaron en diferentes revistas para

${ }^{13}$ REVEL, Jean- François, El renacimiento democrático, trad. Manuel de Lope, Plaza \& Janés/ Cambio 16, Barcelona 1992, pp. 214 y 221. (Le regain démocratique, Librairie Arthème Fayard, 1992).

${ }^{14}$ BELL, Daniel, «Las guerras culturales en U. S. A. (1965- 1990). Comunidad, corrección política y multiculturalismo», Claves de Razón Práctica, nº 33, junio 1993, p. 26.

${ }^{15}$ BELL, Daniel, «Las guerras culturales en U. S. A. (1965- 1990). Comunidad, corrección política y multiculturalismo», p. 26.

${ }^{16}$ Algunos eran nombrados y consultados por Fukuyama: Zbigniew Brzezinski con Between two ages: America's role in the technotronic era; Robert Nisbet con Social change and History; Ian Wilson en su artículo publicado en Problems of comunism: «Leadership by 'lines': China's unresolved succession»; Daniel Bell con The cultural contradictions of capitalism y otros artículos; Jeanne Kirkpatrick (perteneciente al consejo de redacción de The National Interest) con el trabajo publicado en la revista neoconservadora Commentary: «Dictators and double standars»; Saymour Martin Lipset en «Some social requisites of democracy: economic development and political legitimacy», publicado en American Political Social Review; Samuel Huntington en Political order in Changing Sovieties; e Irving Kristol, que sería el editor de la revista The National Interest.

"CUADERNOS DE ESTUDIOS GALLEGOS", Tomo XLVI, Fascículo 111, Santiago 1999. 
la difusión de sus ideas: Commentary, Encounter, New Criterium, American Scholar, Public Opinion y Public Interest. Y buscaron cobijarse en instituciones como: The Hoover Institution, The American Enterprise Institute y The Heritage Foundation ${ }^{17}$.

El término «neoconservador» fue acuñado por Michael Harrington, un liberal en sentido anglo-americano, en $1973^{18}$. Los neoconservadores no poseían un cuerpo ideológico común e incluso todos, menos Irving Kristol, rechazaban el apelativo. Pese a ello, se asociaban, sin demasiados prejuicios, con el neoliberalismo.

Indagando en sus orígenes. Después de la Segunda Guerra mundial, se percibían en Estados Unidos tres corrientes conservadoras parcialmente diferentes. La primera corriente era la de los «libertarios extremos»o «libertarios», contrarios a la expansión estatal y partidarios de ampliar la libertad económica y el individualismo. La segunda tendencia englobaba a los «tradicionalistas», que planteaban el regreso a los principios éticos y religiosos tradicionales ante el relativismo cultural de la izquierda liberal. La tercera inclinación correspondía a los anticomunistas, obsesionados con el expansionismo comunista.

Significativamente, el «neoconservadurismo» no nació de ninguna fuerza o movimiento conservador ya existente. Su formación, se produjo en el seno del movimiento intelectual liberal, que en Estados Unidos se denominaba Liberalismo Radical. Así, salvo Nisbet, todos pertenecían al socialismo americano ${ }^{19}$, incluso, algunos como Irving Kristol habían sido

${ }^{17}$ OLIET PALÁ, Alberto, «Neoconservadurismo», en Vallespín ed., Historia de la Teoría Política, vol. 5, Alianza editorial, Madrid, 1995, p. 399.

${ }^{18}$ Para ésta y sucesivas consideraciones referentes a los «neocons», ver: OLIET PALÁ, Alberto, «Neoconservadurismo», p.399- 489.

${ }^{19}$ Seymour Martin Lipset afirmaba que después de las elecciones de 1976, los Estados Unidos eran la única nación industrializada democrática en la que no ocupaba cargo ni un sólo candidato de un partido laboral o socialista. Y resaltaba que «ello no significa que los estadounidenses no tengan oportunidad de votar por los socialistas. En las elecciones de 1976, candidatos de seis diferentes partidos radicales figuraron en las papeletas electorales de varios estados. Estas incluían al Partido Laboral Socialista, que ha venido presentando candidatos a la Presidencia desde fines de siglo pasado; el Partido Socialista, una fracción minúscula de lo que fue en otro tiempo un partido más grande con ese mismo nombre; el Partido Laboral de los E. U. A., vástago de una facción del grupo de estudiantes en pro de una sociedad democrática; el Partido del Pueblo, un grupo que se define a sí mismo como socialista democrático; el Partido Socialista de los Trabajadores

"CUADERNOS DE ESTUDIOS GALLEGOS", Tomo XLVI, Fascículo 111, Santiago 1999. 
trotskistas. El «neoconservadurismo» era, pues, una retracción endógena dentro del liberalismo.

El verdadero germen del «neoconservadurismo» lo constituyó el famoso debate sobre el «fin de las ideologías» desatado por Daniel Bell ${ }^{20}$.

(trotskista), y el Partido Comunista. Sin embargo ninguno de ellos alcanzó la cifra de 100.000 votos en toda la nación... Este triste expediente de apoyo electoral al socialismo representa algo cercano al punto más bajo, en la larga serie de esfuerzos de más de un siglo, por crear un movimiento socialista en los Estados Unidos. Desde el punto de vista electoral, el esfuerzo que tuvo mayor éxito fue el del Partido Socialista, que antes de la Primera Guerra Mundial contaba con muchos líderes sindicales entre sus 125.000 miembros y que logró la elección de más de 1.000 funcionarios públicos: desde alcaldes y legisladores estatales hasta congresistas nacionales». Lipset finalizaba sus disertaciones sobre el fracaso de los partidos socialistas y laboristas, asegurando que analizando detalladamente la historia norteamericana y su estructura política, se podría llegar a la conclusión de que la poca representatividad de los partidos socialista y laborista se debía a que los dos principales partidos políticos (Demócrata y Republicano) habían perseguido los mismos principios que aquellos partidos en Europa. Con esta nota queda clara la existencia de partidos socialistas en Estados Unidos y queda por aclarar, pese a los esfuerzos de Lipset -un «neocon»-, porqué esos partidos políticos no triunfaron. Ver MARTIN LIPSET, Seymour, «Socialismo en los Estados Unidos», Facetas, vol. 10, $\mathrm{n}^{\circ}$, 1977, pp. 3- 12.

${ }^{20}$ BELL, Daniel, El final de las ideologías, Tecnos, Madrid, 1964 («The end of ideology», Encounter, 5, 1955). Para una mayor profundización en BELL ver: BELL, D., Las Ciencias Sociales desde la II Guerra Mundial, Alianza, Madrid, 1984; BELL, D., Las contradicciones culturales del capitalismo, Alianza, Madrid, 1977 (The cultural contradictions of capitalism, Heinemann, London, 1976). En España también tuvo su propia versión del final de las ideologías unos años después, con una gran literatura crítica en la época, se trata del libro de FERNANDEZ DE LA MORA, Gonzalo, El Crepúsculo de las ideologías, Salvat- Alianza Editorial, Navarra, 1971 (Rialp, Madrid, 1965). En ese libro, González de la Mora defendía, en un trabajo de mucho más peso teórico que empírico, que el crepúsculo de las ideologías llevaba a una nueva teoría del Estado: la ideocracia o logoarquía. Pronosticaba la sustitución de las ideologías por las ideas rigurosas, adecuadas y concretas, y que la ideocracia o logoarquía es el Estado del futuro. Todo en base a una definición de ideología como «filosofía política, simplificada, generalizada, dramatizada, sacralizada y desrealizada; en suma: un subproducto mental, una pseudoidea, una razón caricaturizada y corrompida por un intenso y sostenido tratamiento de masificación» (FERNÁNDEZ 1971: 153). Llegaba a la idea de «ideocracia» por el derrumbamiento de las ideologías, fundamentados en cinco puntos: 1- El abstencionismo electoral, la decadencia del partidismo y el entusiasmo, la atrofia de la prensa doctrinaria, la despolitización del ocio y la deshumanización del Estado son causas y efectos del desinterés ciudadano por la política. 2-Cuando dos ideologías contrapuestas tienden a fundirse es que están en trance de disolución. 3- La ética social ha alcanzado un alto nivel de perfección teórica. 4- Depuración, despragmatización y despolitización de las religiones. 5- El desarrollo económico solidariza, intelectualiza, responsabiliza y dignifica a los individuos y homogeneiza las clases y los intereses.

"CUADERNOS DE ESTUDIOS GALLEGOS", Tomo XLVI, Fascículo 111, Santiago 1999. 
En esta tesis sostenía, de una forma sumaria, que las propuestas, los ideales y las normas éticas con pretensiones universalistas se habían diluido en el acontecer pragmático de las sociedades. Los sucesos de 1968 destaparon las limitaciones de la tesis de Bell. Y esos acontecimientos del 68 hicieron que Bell en Las contradicciones culturales del capitalismo afirmara que el agotamiento de las ideologías llevaría a buscar otras nue$\operatorname{vas}^{21}$. Esta teoría dejaba traslucir una autocomplacencia liberal y una confianza en el Estado pluralista y socializante.

Pero las circunstancias cambiaron, y en los años setenta surgía una coyuntura histórica y económica que exigió un activismo intelectual fuerte, abanderado por los «neocons». A saber: vuelta al mercado, defensa a ultranza del capitalismo y puesta en duda del Estado social. Esta situación surgió por la revuelta estudiantil de los Estados Unidos, los problemas con los «ghetos», la derrota de Vietnam, la procedencia judía de muchos «neoconservadores» (obligados a poner en duda los postulados de la «nueva izquierda» en el estado de Israel, la cual despreciaba los valores liberales) y la nominación del presidente demócrata McGovern, que les hizo posicionarse en favor de Nixon (sobre todo Kristol).

Tanto Daniel Bell como Irving Kristol y otros muchos pertenecieron en un principio a la saga de los «New York Intelectuals» ${ }^{22}$, quienes defendían un anticomunismo producto de su antiestalinismo; una ambigüedad

${ }^{21}$ Alberto Oliet pensaba que el artículo de Francis Fukuyama «¿el final de la historia?» (Oliet se olvidaba de la interrogación y de «H» mayúscula) hacía cristalizar la impotencia definitiva de la Ideología para motivar la acción política. Ver OLIET PALÁ, Alberto, «Neoconservadurismo», p. 418.

${ }^{22}$ Los «New York Intelectuals» fueron el símbolo de la transformación cultural y social de la América de Posguerra. En su mayoría son judíos que se integran en una sociedad W.A.S.P. (White Anglo Saxon Protestant) e intentan integrar la cultura europea en un contexto plenamente americano. Algunos de los principales integrantes de esta saga fueron: el crítico literario Philip Rabv (editor de Partisan Review); el ensayista Lionel Trilling; Alfred Kazin, Susan Sontang o Robert Silver, estos últimos ensalzados por The New York Review of Books (que nace en 1963 fusionando las ideologías de la alta sociedad W.A.S.P. y los intelectuales de New York), el medio literario más influyente de este grupo; los poetas Delmore Schwartz, Allen Ginsberg, Hohn Berryman o Robert Lowell; los críticos de arte Clement Greenberg y Harold Rosenberg; el filósofo político anticomunista Sidney Hook; el gran sacerdote de la socialdemocracia americana Irving Howe; Daniel Bell; Irving Kristol; los analistas multirraciales Nathan Glazer y Daniel Moyniban; y novelistas como Norman Mailer, Bernad Malamud o Philip Roth. Ver PINTO, Diana, «La saga de los New York intellectuals», Debatts, $\mathrm{n}^{\circ} 16$, p. 53-54.

"CUADERNOS DE ESTUDIOS GALLEGOS", Tomo XLVI, Fascículo 111, Santiago 1999. 
respecto a la tradición política liberal de las élites W.A.S.P. (White Anglo Saxon Protestant- Blanco, Anglosajón y Protestante-); una desconfianza de la américa auténtica percibida como antiintelectual, hostil y potencialmente peligrosa; y una tensión existencial entre la fidelidad a sus raíces críticas y la satisfacción de verse asimilados social y económicamente al cogollo de la vida americana ${ }^{23}$. La mayoría de estos «New York Intellectuals» son inmigrantes judíos dela Europa del Este. La evolución de esta saga intelectual comenzó con el radicalismo revolucionario de los años 30 cuando cambian su tendencia izquierdista prosoviética gracias a Stalin. Más tarde encarnarían el triunfo del liberalismo crítico de comienzos de los años 60 y el radicalismo exacerbado de los años 60, hasta llegar a la moderación conservadora y neoconservadora de los años 70. Esto fue debido a que algunos de los «New York Intellectuals» se sienten traicionados por los movimientos que ellos mismos habían jaleado, como las revueltas estudiantiles contra Vietnam. Y otros ven en estas protestas un acto peligroso, y se sienten más cerca del poder. Ante estos hechos, todos los intelectuales próximos a la revista Commentary (encabezados por Norman Podhoretz y Nathan Glazer) y los cercanos a la revista National Review (Irving Kristol y Daniel Bell) se convertirían en intelectuales pragmáticos y realistas, abriéndose al neoconservadurismo. De tal forma que la revista Commentary se convirtió en el centro del neoconservadurismo en nombre de una visión ultrajudía y sionista del mundo y las relaciones internacionales ${ }^{24}$. Este grupo sólo fue un botón de muestra del fracaso de la saga de los intelectuales «made in New York», quienes se vieron engullidos por el pragmatismo de la democracia americana. Curiosamente Irving Kristol es miembro de la revista The National Interest, revista conservadora de Ciencia Política y Relaciones Internacionales donde se publicó el famoso artículo de Fukuyama «The end of History?».

Abandonando su origen, los «neocons» diagnosticaban una preocupante «ingobernabilidad» en los Estados Unidos de los años 70. Se centraban en:

\footnotetext{
${ }^{23}$ PINTO, D., «La saga de los New York intellectuals», p. 54.

${ }^{24}$ PINTO, D., «La saga de los New York intellectuals», p. 60-63.
}

"CUADERNOS DE ESTUDIOS GALLEGOS", Tomo XLVI, Fascículo 111, Santiago 1999. 
a. La impotencia estatal para responder a la multiplicidad y entidad de las expectativas del ciudadano medio.

b. Crisis del Estado al generar unas expectativas en la población que no podía satisfacer.

c. Crisis moral y espiritual por la vacuidad de valores y educación corrompida.

Frente a esto, proponían:

a. Reducción de las responsabilidades del estado (recortes sociales, privatización...).

b. Moderación en el ensalzamiento de la democracia.

c. Renovación moral y espiritual basada en la tradición y la jerarquía.

El padre filosófico del «neoconservadurismo» fue el filósofo Político de la Universidad de Chicago Leo Strauss (1899-1973). Strauss atacó el subjetivismo de la modernidad y enseñó que las ideas fundacionales de virtud y excelencia debían encontrarse en los escritos políticos clásicos. Atrajo a un gran número de discípulos y exégetas que ocuparon posiciones clave en la rama ejecutiva de la Administración republicana. Su discípulo más famoso fue Allan Bloom, también de la Universidad de Chicago, quien tuvo un gran éxito con su libro The closing of the american mind. Francis Fukuyama está influenciado por Bloom ${ }^{25}$, y por tanto, estrechamente vinculado, e influenciado, por Strauss ${ }^{26}$.

Intelectualmente, Strauss se formó en la Alemania de Weimar en Filosofía y Teología (era judío). El Filósofo Político centró parte de su obra en hacer confluir la religión y la filosofía política. Ambas se practicarían

\footnotetext{
${ }^{25}$ Fukuyama utiliza la traducción de Bloom de La República de Platón en sus escritos. Esta obra es de capital importancia en su tesis sobre «el fin de la Historia».

${ }^{26}$ BELL, Daniel, «Las guerras culturales en U. S. A.. Comunidad, corrección política y multiculturalismo», p. 26.
}

"CUADERNOS DE ESTUDIOS GALLEGOS", Tomo XLVI, Fascículo 111, Santiago 1999. 
en la escritura de dos formas: «esotérica» y «exotéricamente» ${ }^{27}$. La «exotérica» estaría centrada en lo que el autor transmitiría al lector vulgar, y la «esotérica» sería el sentido último del texto, únicamente accesible para iniciados.

Por otro lado, Strauss estudió con minuciosidad la tensión entre los autores antiguos y los modernos, haciendo, a su vez, una crítica al discurso filosófico de la modernidad ${ }^{28}$. Esta crítica a la modernidad, era debida a su elección personal. Strauss, como judío ${ }^{29}$, había optado por la no integración de los judíos y la creación de un Estado hebreo en Israel. La filosofía política liberal pretendía la universalidad y la unificación del hombre bajo principios abstractos, y Strauss defendía algo, el sionismo, que se apoyaba en el «particularismo» de ser el pueblo elegido ${ }^{30}$.

La obra culmen de Strauss fue Natural Right and History ${ }^{31}$. Y en ésa, como en muchas otras obras, mantenía una actitud de priorizar el estudio

${ }^{27}$ Joseph McCarney notaba la distinción entre discurso «esotérico»y «exotérico» en Fukuyama. Ver McCARNEY, Joseph, «Endgame», Radical Philosophy, 62, Autumn, 1992, pp. 35- 38.

${ }^{28}$ Esto lo hacía notar Fukuyama cuando aludía a la correspondencia entre Kojève y Strauss que aparecía en la edición de 1991 del libro On Tyranny: FUKUYAMA, Francis, «Reflections on the end of History, five years later», After History? Francis Fukuyama and his critics, pp. 239- 257.

${ }^{29}$ Sobre este particular ver: DEUTS, Kenneth and NICGORSKI, Walter, Political philosopher and jewish thinker, Rowmen \& Littlefield Publischers, 1994.

${ }^{30}$ VALLESPÍN, Fernando, «La vuelta a la tradición clásica: Leo Strauss, E. Voegelin», en Fernando Vallespín ed., Historia de la Teoría Política, vol. 5, Alianza editorial, Madrid, 1995, p. 364.

${ }^{31}$ STRAUSS, Leo, Natural rights and History, University of Chicago Press, 1953. En este autor son escasas las traducciones al castellano, lo que limita considerablemente su difusión. Otras obras de Strauss son: STRAUSS, L., Spinoza's critique of religion, Schocken Books, New York, 1965; STRAUSS, L., Persecution and the art of writing, Free Press, Glencoe, 1952; STRAUSS, L., Thoughts on Machiavelli, Free Press, Glencoe, 1958 (Meditación sobre Maquiavelo, trad. Carmen Gutierrez de Caba, Instituto de Estudios Políticos, 1964; STRAUSS, L. What is political philosophy?, Free Press, Glencoe, 1959; STRAUSS, L., The city and man, Rand MacNally, Chicago, 1964; STRAUSS, L., Studies in platonic political philosophy, Universidad de Chicago Press, Chicago, 1983; STRAUSS, L., On Tyranny, Free Press, Glencoe, 1963; STRAUSS, L. and CROPSEY, J. (eds.), History of political philosophy, University of Chicago Press, 1963; STRAUSS, L., Socrates and Aristophanes, Schoocken, New York, 1966; STRAUSS, L., Liberalism: ancient and modern, Basic Books, New York, 1968; STRAUSS, L., Xenophon's Socrates, Ithaca, 1972; STRAUSS, L., The argument and the action in Plato's laws, Chicago 
de los clásicos griegos y otros grandes filósofos de otras épocas, que para él eran la génesis de la Filosofía Política. Para Strauss existían las verdades en teoría política al margen del tiempo y lugar (era un gran antihistoricista). Decía que los textos debían estudiarse por sí solos, porque representaban los problemas de todas las épocas ${ }^{32}$ : Strauss concebía como investigación histórica el estudio literal de los textos en busca de la intención última del autor.

Otras ideas de Strauss $^{33}$ fueron: su concepción de la educación ajena a la cultura de masas, declaraba la «crisis de Occidente» como la crisis de la modernidad, decía que el mundo Occidental estaba amenazado por el comunismo y el despotismo Oriental y que la democracia liberal se hallaba en decadencia ${ }^{34}$.

Con la relación ideológica, política y de escuela intelectual queda suficientemente explicado el contexto de Fukuyama. De otro modo, Fukuyama es un norteamericano de tercera generación ${ }^{35}$ de origen japonés ${ }^{36}$, na-

University Press, Chicago, 1975; STRAUSS, L., The rebirth of classical political rationalism. Essays and lectures by Leo Strauss, essays and lectures selected and introduced by Thomas L. Plange, University of Chicago Press, Chicago, 1989. Para una bibliografía más amplia del autor véase: STRAUSS, L., Studies in platonic political philosophy, T. Pangle, ed., University of Chicago Press, 1983; STRAUSS, L., Faith and political philosphy: the correspondence between Leo Strauss and Eric Voegelin, University Park, Pennsylvania, 1993.

32 TUCK, Richard, «Historia del pensamiento político», en Peter Burke Formas de hacer Historia, Alianza Universidad, Madrid, 1994, p. 244- 248.

${ }^{33}$ Una importante influencia para Fukuyama, Allan Bloom, también se encargó de Strauss: BLOOM, Allan, «Leo Strauss: september 20, 1899- october 18, 1973», Political Theory, 2, 1974, pp. 381- 387. Para otras obras sobre Strauss, ver: MEIER, Heinrich, Carl Schmitt, Leo Strauss et la notion de politique: un dialogue entre absents. Suivi du commentaire de Leo Strauss sur la notion de politique. Et le trois letters inédites á Carl Schmitt des années 1932-33, Commentaire/ Juilliard, París, 1990; LAMPERT, Laurence, Leo Strauss and Nietzsche, University of Chicago Press, Chicago, 1996.

${ }^{34}$ VALLESPÍN, Fernando, «La vuelta a la tradición clásica», pp. 372- 379.

${ }^{35}$ CRUZ, Juan, «La erupción de un volcán llamado Fukuyama», en «Babelia», $E l$ País, 7 de marzo, 1992, p. 18. Además, Cruz añadía en referencia al paso del norteamericano por España que había estado «frío y veloz como un corredor de motos... monocorde en su tono de conversación, impasible, este norteamericano de tercera generación parece el japonés que tiene dentro: exacto, milimétrico...».

${ }^{36}$ Ver HALLIDAY, Fred, «An encounter with Fukuyama», New Left Review, 193, mayo- junio, 1992, p. 89.

"CUADERNOS DE ESTUDIOS GALLEGOS", Tomo XLVI, Fascículo 111, Santiago 1999. 
cido en Chicago en $1952^{37}$. Hijo de un clérigo protestante que luego fue profesor de Religión. Se crio en New York y se educó en Yale, La Sorbona y Harvard, estudiando Literatura Comparada y Ciencias Políticas, con maestros como Allan Bloom, Paul de Man, Rolan Barthes y Jacques Derrida $^{38}$, y se doctoró en Filosofía y Letras ${ }^{39}$, concretamente en Ciencia Política en la Universidad de Harvard ${ }^{40}$ en el «Deparment of Gobvernment» sobre «Soviet Foreign Policy, Middle Eastern Politics» y con la tesis sobre relaciones internacionales «Soviet threats to intervene in the middle easat, 1956-1973» bajo la supervisión del profesor Nadav Safran en 1981. También en los años 1981 y 82 perteneció al Departamento de Estado y fue miembro de la delegación de las conversaciones entre Egipto-Israelí sobre la autonomía de Palestina.

Durante este tiempo trabajó en la Rand Corporation haciendo informes sobre asuntos concretos y prácticos de política internacional ${ }^{41}$, hasta que en el año 1989 accedió al puesto de director adjunto de planificación política en el Departamento de Estado ${ }^{42}$, donde estuvo hasta 1990. Actualmente es «Hirst Professor» de Política Pública en la George Mason University en Fairfax (Virginia) ${ }^{43}$, director del International Transactions Program y también es miembro investigador de la Rand Corporation en Washington D. C. ${ }^{44}$. Asimismo forma parte de la John Hopkins University School for Advanced International Studies Foreign Policy Institute, don-

${ }^{37}$ FUKUYAMA, Francis, EL final de la Historia y el último hombre, solapa interior del libro. Esto era confirmado por CRUZ, Juan, «Entrevista con Fukuyama», El País, 11 de diciembre de 1991, p. 7. Cruz afirmaba que en aquel momento, Fukuyama tenía treinta y nueve años.

${ }^{38}$ MORA-FIGUEROA, Santiago de, «El acabose», Nueva Revista, ${ }^{\circ} 1$, febrero, 1990, p. 45. Mora- Figueroa añadía en la misma página que "le aburrieron los estructuralistas parisinos".

${ }^{39}$ FUKUYAMA, Francis, El final de la Historia y el último hombre, solapa interior del libro.

40 «Francis Fukuyama», World of Penguin, http://www.puffin.co.uk:80/Penguin/ Authors/814.html.

${ }^{41}$ Es autor de numerosos artículos sobre los asuntos exteriores soviéticos y rusos, de Relaciones Internacionales y Política Económica. BURNS, Timothy ed. After History?. Francis Fukuyama and his critics, p. 264.

${ }^{42}$ Como él mismo constataba en su artículo original de 1989: FUKUYAMA, Francis, «The end of History?», p. 3

${ }^{43}$ «Francis Fukuyama», World of Penguin.

${ }^{44}$ «Francis Fukuyama», World of Penguin.

"CUADERNOS DE ESTUDIOS GALLEGOS", Tomo XLVI, Fascículo 111, Santiago 1999. 
de además es director del proyecto de telecomunicaciones ${ }^{45}$. Es un científico político especializado en asuntos político militares del Medio Este y de Política Exterior de la antigua Unión Soviética ${ }^{46}$.

Precisamente su primer libro está referido a la Unión Soviética The third world: the last three decades ${ }^{47}$, del cual es editor junto a Andrzej Korbonski. Sobre este mismo tema publicaría diferentes informes para la Rand Corporation como «The Soviet Union and Iraq since 1968» y «The security of Pakistan: a trip report» en 1980; «The soviet threat to the Persian Gulf» en 1981; «Moscow's post-Brezhnev reassessment of the third world» en 1986, «Soviet civil-military relations and the power projection mission» en 1987; «Gorbachev and the new societ agenda in the third world» en 1989. Por otro lado, también publicó diferentes artículos referentes al tema como «The USSR and the middle east» ${ }^{48}$, «Pakistan since the soviet invasion of Afghanistan ${ }^{49}$, «New directions for Soviet middle policy: implications for the Atlantic aliance ${ }^{50}$, «The new marxist leninist states and internal conflict in the third world $»^{51} \mathrm{O}$ «The political character of the overseas empire ${ }^{52}$ entre otros. También se dedicó a Asia en «Asia in a global war» ${ }^{53}$.

Después del éxito y la polémica de su artículo «The end of History?» y de la contestación a sus exégetas «A reply to my critics», ambas publicadas en la revista The National Interest, dimitió de su cargo político y volvió a la Rand Corporation ${ }^{54}$, donde escribió The end of History and

\footnotetext{
45 «Francis Fukuyama», en «Speakers and panelist», Pcforum, 1996 http:// www.edventure.com:80/pcforum/96pcf/speakers/fukuyama.html.

${ }^{46}$ «Francis Fukuyama», en «Speakers and panelist», Pcforum, 1996.

${ }^{47}$ Cornell University Press, Ithaca- New York, 1987.

${ }^{48}$ Middle East Contemporary Survey, vol. IV, 1981.

${ }^{49}$ en TAHIR-KHELI, SHIRIN (ed), U.S. Strategic interest in southwest Asia, praeger, 1982.

${ }^{50}$ en SPIEGEL, Steven L., The middle east and the western alliance, George Allen and Unwin, 1982.

${ }^{51}$ en RA'ANAN, Uri et al., Third world marxist-leninist regimes: strengths, vulnerabilities, and U.S. Policy, Institute for Foreign Policy Analysis Special Report, 1985.

${ }^{52}$ en ROWEN, Henry and WOLF, Charles (eds.), The future of the soviet empire, New York, St. Martin's Press, 1987.

${ }^{53}$ en Comparative Stategy, vol. 6, n. 4, 1987.

${ }^{54}$ MORA FIGUEROA, Santiago de, «Postdata al acabóse», Nueva Revista, $\mathrm{n}^{\circ} 2$, marzo 1990, p. 31. A su vez Fukuyama declaraba que la Rand Corporation le había
} 
the last $\mathrm{man}^{55}$. Dos años después, fue invitado por el politólogo Timothy Burns en el libro After History? Francis Fukuyama and his critics, para hacer un último intento de aclarar los mal entendidos de la tesis del «fin de la Historia». El artículo se tituló «Reflections on the end of History, five years later» y también fue reproducido por la revista History and Theory. Por último, sin abandonar la Rand Corporation ${ }^{56}$, escribió el libro Trust: the social virtues and the creation of prosperity ${ }^{57}$ sobre el cual ha habido otro debate internacional y donde se ven más claras las posturas políticas e intelectuales de Fukuyama. Aparece como alguien comprometido con el estado, con la sociedad civil y con la importancia de la cultura. Quizá, como escribe el propio Fukuyama, todo el revuelo levantado alrededor de The end of History and the last man fuera un malentendido; o quizá el no se expresó del todo bien y las afirmaciones le encorsetaban en una etiqueta que no era realmente la suya. Después de este último libro se ha publicado otros trabajos como «On the possibility of writing a Universal History ${ }^{58}$.

dejado un despacho para elaborar el libro: FUKUYAMA, Francis, EL fin de la Historia y el último hombre, p. 9.

${ }^{55}$ Libro con el cual ganó los premios Premio Capri en Italia y el galardón de Los Angeles Times' book critics award. Ambos en 1992.

${ }^{56}$ Fukuyama no abandonó la Rand Corporation desde 1989 hasta nuestros días. Ver FUKUYAMA, Francis, «El capital social y la economía mundial», Política Exterior, vol. IX, n'. 47, octubre/noviembre 1995, p. 77.

${ }^{57}$ Además de estas obras, Fukuyama empezó su carrera intelectual ocupándose de Afghanistan: FUKUYAMA, Francis, The future of the soviet role in Afghanistan, a trip report, Santa Mónica, Rand Corporation, 1980; véase VERCELLIN, Giorgio, «Fine della storia, storia orientale e orientalistica», Studi Storici, julio- septiembre1991, pp. 97- 110. Hasta 1989 hizo estudios sobre los Estados del Tercer Mundo con orientación socialista como Ethiopia, Mozambique y Yemen del sur; ver HALLIDAY, Fred, «An encounter with Fukuyama», New Left Review, n 193, mayo-junio, 1992, p. 90. Otro trabajo fue: FUKUYAMA, Francis, «Capitalism and democracy: the missing link», Journal of Democracy, $\mathrm{n}^{\circ} 3$, julio 1992, pp. 100-110; ver FUKUYAMA, Francis, «Reflections on the end of History, five years later» p. 257. Otras publicaciones son FUKUYAMA F., KONGDAN, OH, «The US- Japan security relationship after the cold war», Rand Paper, septiembre 1993; FUKUYAMA, F., «Great planes», New Republic, 1993, pp. 10-11; FUKUYAMA F., «Inmigrants and family values», Commentary, n ${ }^{\circ} 95,1993$, pp. 26-32; FUKUYAMA, F., «The primacy of culture», Journal of Democracy, n6, 1995, pp. 7-14.

${ }^{58}$ en MELZER, Arthur M., WEINBERGER, Jerry and ZINMAN, Richard (eds), History and the idea of progress, Ithaca- New York, Cornell University Press, 1995.

"CUADERNOS DE ESTUDIOS GALLEGOS", Tomo XLVI, Fascículo 111, Santiago 1999. 